**TITLE**

ASP Conference Series, Vol. **VOLUME ${ }^{* * *}$, **YEAR OF PUBLICATION**

**NAMES OF EDITORS**

\title{
Gamma rays from clusters of galaxies
}

\author{
Pasquale Blasi \\ INAF/Osservatorio Astrofisico di Arcetri \\ Largo Enrico Fermi, 5 \\ I-50125 Firenze (ITALY)
}

\begin{abstract}
The search for gamma radiation in clusters of galaxies represents a precious tool to investigate the history of these large scale structures. Clusters or sources within them accelerate cosmic rays, as demonstrated by the detection of radio halos, hard X-rays and UV emission, and confine them over cosmological time scales. Nonthermal and thermal phenomena may be closely related and observations of gamma rays may tell us about this link. In this paper we review the physics of cosmic ray acceleration and confinement in clusters of galaxies and the related gamma ray signatures. In particular we describe in some detail the role of cluster mergers for the acceleration of nonthermal particles. The perspectives for gamma ray detection with GLAST and with ground based detectors are also discussed.
\end{abstract}

\section{Introduction}

The presence of nonthermal particles in clusters of galaxies is a well established fact. These particles are responsible for extended synchrotron radio halos in several clusters (see Feretti et al., 2000 for a recent rewiew), as well as for hard X-ray (HXR) and extreme ultraviolet (EUV) excesses (see e.g. Fusco Femiano et al., 1999, 2000; Lieu et al., 1996). Several explanations have been proposed for the origin of this radiation, but at present there is no conclusive evidence in favor or against any of these models.

The simplest explanation for the HXR excess is based on the inverse compton scattering (ICS) of the same relativistic electrons that are responsible for the radio halos. In this case, low values of the intracluster magnetic field are required, that seem in some contradiction with the much larger values of the fields evaluated through faraday rotation measurements (RM) (Eilek 1999; Clarke, Kronberg \& Böringer 1999). These measurements are however quite difficult, and the discrepancy needs to be considered critically. The severe energy losses associated with the relativistic electrons make the nonthermal phenomena due to synchrotron and ICS of relativistic electrons transient phenomena, with duration not longer than a few billion years. However, if the acceleration processes occur during some violent phenomenon such as cluster mergers, some level of reacceleration may be expected due to the presence of turbulence in the intracluster medium (Brunetti et al. 2001a, 2001b). 
Alternative explanations of the HXR excess have also been proposed, based on acceleration of electrons from the thermal bath and bremsstrahlung radiation from these particles (Ensslin, Lieu \& Biermann 1999; Blasi 2000; Dogiel 2000; Sarazin \& Kempner 2000). These models also have their drawbacks, as discussed by Petrosian (these proceedings) and Petrosian (2001).

An important theoretical insight transformed our way of looking at clusters: cosmic rays accelerated in clusters are trapped there for cosmological times (Berezinsky, Blasi, \& Ptuskin 1997; Völk, Aharonian, \& Breitschwerdt 1996). Clusters behave as cosmological storage rooms for cosmic rays. The combination of this argument and the ever-increasing mass of observations of nonthermal phenomena, generated an unprecedented interest in clusters as possible sources of gamma rays. The detection (or not) of gamma radiation by one of the future gamma ray telescopes such as GLAST, or even current ground based telescopes such as STACEE or HEGRA would allow us to weigh the nonthermal content of clusters and achieve a better understanding of the nonthermal history of these large scale structures.

The issue of nonthermal radiation is clearly related to the problem of acceleration of particles: although the common wisdom is that the acceleration occurs during mergers of subclusters, there are several arguments which complicate this simple picture. We discuss this important issue at length in this review.

Throughout the paper we assume a flat cosmology $\left(\Omega_{0}=1\right)$ with $\Omega_{m}=0.3$, $\Omega_{\Lambda}=1-\Omega_{m}=0.7$ and a value for the Hubble constant of $70 \mathrm{~km} / \mathrm{s} / \mathrm{Mpc}$.

The paper is planned as follows: in $\S 2$ we discuss the physics of cosmic ray confinement in clusters of galaxies; in $\S 3$ we summarize the gamma ray predictions for gamma rays from clusters of galaxies. $\S 4$ is devoted to the investigation of merger shocks as cosmic ray accelerators. The consequences of gamma ray production from clusters onto the diffuse gamma ray background are discussed in $\S 5$, while our conclusions are presented in $\S 6$.

\section{Cosmic Ray confinement. When $\gamma$-rays became an option}

The bulk of high energy particles in clusters of galaxies propagate diffusively. The diffusion time scale for a particle with energy $E$ on a spatial scale $R$ comparable with the size of a cluster is

$$
\tau_{\text {diff }} \approx \frac{R^{2}}{4 D(E)}
$$

where $D(E)$ is the diffusion coefficient. It is easy to see that the energy at which the diffusion time becomes shorter than the age of the universe is

$$
\tilde{E}=2 \times 10^{8} B_{\mu} \mathrm{GeV}
$$

for a Bohm diffusion, and

$$
\tilde{E}=3 \times 10^{4} B_{\mu} L_{20}^{-2} \mathrm{GeV}
$$

for a Kolmogorov spectrum of the fluctuations on the magnetic field $B_{\mu}$ (in $\mu G) . L_{20}$ represents here the scale where there is most of the power in the Kolmogorov spectrum, in units of $20 \mathrm{kpc}$. These expressions for $\tilde{E}$ tell us that the 
bulk of cosmic rays is confined within clusters, which therefore behave as cosmological storage rooms for cosmic rays (Berezinsky, Blasi \& Ptuskin 1997; Völk, Aharonian, \& Breitschwerdt 1996). Any process or any source that accelerates particles within the cluster volume contributes to increase the nonthermal content of the intracluster gas. The present value of the energy density of cosmic rays in a cluster is the result of all these processes integrated over the lifetime of the cluster (comparable to the age of the universe $t_{0}$ ).

This argument is of special importance for particles whose energy losses occur on time scales longer than $t_{0}$, in particular high energy protons (or nuclei). Relativistic electrons with $\gamma>300$ lose energy on time scales shorter than $t_{0}$, so that their energy is radiated away through synchrotron and ICS emission. The fate of these high energy electrons is to finally pile up at lorentz factors around $\sim 100$ where the timescale for losses, dominated now by Coulomb scattering, becomes of several billion years.

For protons, the main channel of energy losses is provided by inelastic proton-proton scattering, with inclusive cross section $\sigma_{p p} \sim 3 \times 10^{-26} \mathrm{~cm}^{2}$. In a cluster of galaxies, the timescale associated with this process is

$$
\tau_{p p}=\frac{1}{n_{\text {gas }} \sigma_{p p} c}=3.5 \times 10^{10} n_{-3}^{-1} \mathrm{yrs},
$$

where $n_{-3}=n_{\text {gas }} / 10^{-3} \mathrm{~cm}^{-3}$ and $n_{\text {gas }}$ is the gas density in the intracluster medium. Inelastic $p p$ scattering is weak enough to allow the accumulation of protons over cosmological times, as discussed above, but also efficient enough for the continuous production of pions, which in turn decay into gamma rays (for neutral pions), electron-positron pairs and neutrinos (for charged pions). The decay chain is as follows:

$$
\begin{gathered}
p+p \rightarrow \pi^{0}+\pi^{+}+\pi^{-}+\text {anything } \\
\pi^{0} \rightarrow \gamma \gamma \\
\pi^{ \pm} \rightarrow \mu+\nu_{\mu} \quad \mu^{ \pm} \rightarrow e^{ \pm} \nu_{\mu} \nu_{e} .
\end{gathered}
$$

The role of electron-positron pairs from $\pi^{ \pm}$decays is subject of much debate and investigation (see also talk by Brunetti, these proceedings). In fact synchrotron emission from these pairs may well reproduce the general features of the radio halos and their diffuse appearance (Colafrancesco \& Blasi 1998), without invoking any additional reacceleration processes. Reacceleration is instead required in radio halo models based on the acceleration of primary electrons and their propagation in the cluster volume. In order to accomodate the HXR emission observed from the Coma cluster, this model requires a magnetic field of $\sim 0.1 \mu G$. This conclusion actually holds for any other model, with the possible exception of models in which a cutoff in the electron spectrum is tuned up in order to reduce the corresponding synchrotron emission. In these cases the magnetic field can be as high as $0.3-0.4 \mu G$ (Brunetti et al. 2001a). It was shown by Colafrancesco \& Blasi (1998) that for the Coma cluster, in the context of the secondary electron model, small magnetic fields imply an overproduction of gamma radiation compared to the EGRET upper limit (Sreekumar et al. 1996). This conclusion may possibly be avoided only if the emission regions of HXRs and radio radiation are different. A careful investigation of all 
these effects is being carried out by Blasi, Brunetti \& Gabici (2002), in order to understand under which conditions, if any, the fine structure of radio halos (spatial distribution of the radiation, spectral steepening in the outer regions of clusters, radio halo statistics) can be accomodated within the so-called secondary electron model (Dennison 1980, Colafrancesco \& Blasi 1998, Blasi \& Colafrancesco 1999), where all the electrons responsible for the radio halo are due to $p p$ scatterings. This investigation is extremely important even if the bulk of the observed nonthermal radiation had to be generated mainly by something other than the secondary $e^{+} e^{-}$pairs. In fact, if to take our own Galaxy as a template of cosmic ray behaviour, it seems likely that in clusters, as well as in the Galaxy, protons outnumber electrons (at least around $1 \mathrm{GeV}$ ) by about a factor 100. The questions then become: where are these protons? And how can we detect them? The answer to these questions is, we believe, in gamma ray observations, both in the GeV range, with GLAST, and in the TeV range, with ground based detectors. In the next section we describe the current predictions of gamma ray fluxes and their physical information load.

\section{3. $\gamma$-ray emission}

A useful way of discussing the gamma ray emission from clusters of galaxies is by simply parametrizing the proton abundance in clusters as a fraction of the thermal (virial) energy of the cluster, or, in other words, in terms of deviations from equipartition. In fig. 1, we plot the gamma ray fluxes expected from a Coma-like cluster as due to $p p$ inelastic scattering, and neglecting at this stage any other contribution. The energy density of cosmic ray protons is taken to be equal to the thermal energy density. In the upper panel we assumed that cosmic rays are injected by a point source (for instance a radio Galaxy) in the center of the cluster, while in the bottom panel cosmic rays are assumed to be injected homogeneously in the cluster volume. The EGRET upper limit and the sensitivity curves for GLAST and for some ground based experiments are also reported in the figure. Some comments are in order: 1) the fluxes of gamma rays above $100 \mathrm{GeV}$ are already at the EGRET sensitivity level, and will certainly be at hand for GLAST. 2) In the presence of protons, it is basically unavoidable to have gamma ray production up to at least the $\mathrm{TeV}$ range; in this energy region, the radiation spectra reproduce the spectrum of parent protons.

It seems that even with present experiments, such as STACEE, it would be possible to put interesting upper limits on the gamma ray fluxes from some nearby clusters of galaxies, such as Coma (Blasi 1999). If the clusters are too far away, then absorption effects due to photon-photon pair production on the cosmic infrared background and smaller fluences may make the detection more difficult or even impossible at energies in excess of a few $\mathrm{TeV}$.

In order to have a more complete picture of the processes that contribute to the gamma ray brightness of a cluster, we need to include at least two other components, namely primary and secondary electrons. Blasi (2000) has carried out this calculation for the case of a merger shock as accelerator, but the basic features remain valid even for a different type of accelerator. A similar numerical calculation was carried out by Miniati et al. (2001).

For a Coma-like cluster, the flux of gamma radiation is plotted in fig. 2 . The 

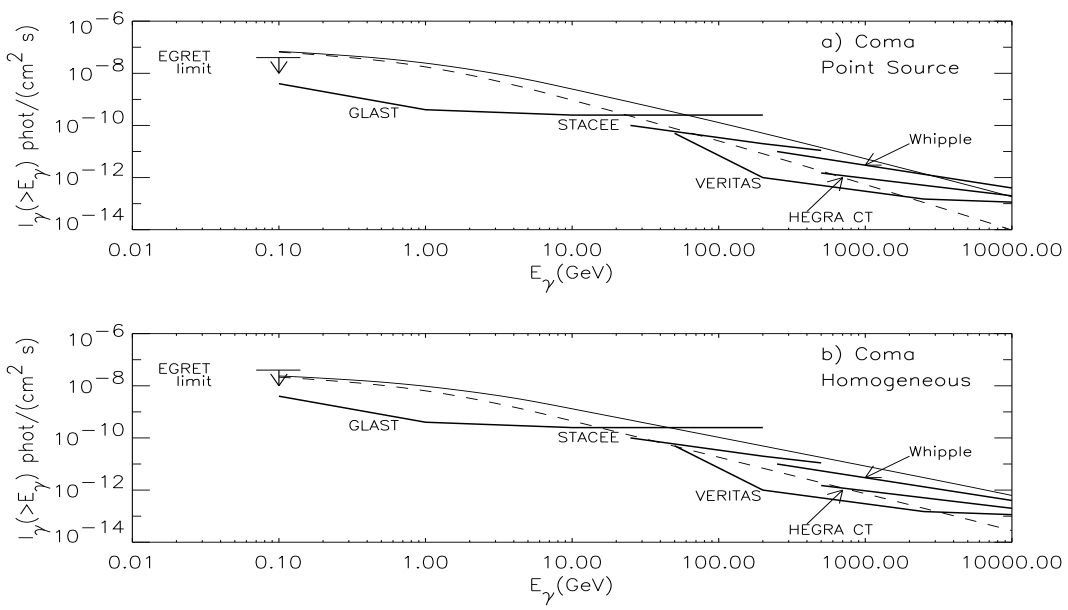

Figure 1. Predicted gamma ray flux from the Coma cluster as produced by $\pi^{0}$ decay (Blasi 1999). The injection spectrum of protons is $E^{-2.1}$ for the solid line and $E^{-2.4}$ for the dashed line. In both cases cosmic rays are assumed in equipartition with the thermal energy. The sensitivities of some present and future gamma ray experiments are also plotted. The injection occurs from a point source in the cluster's center (upper panel) or homogeneously (lower panel).

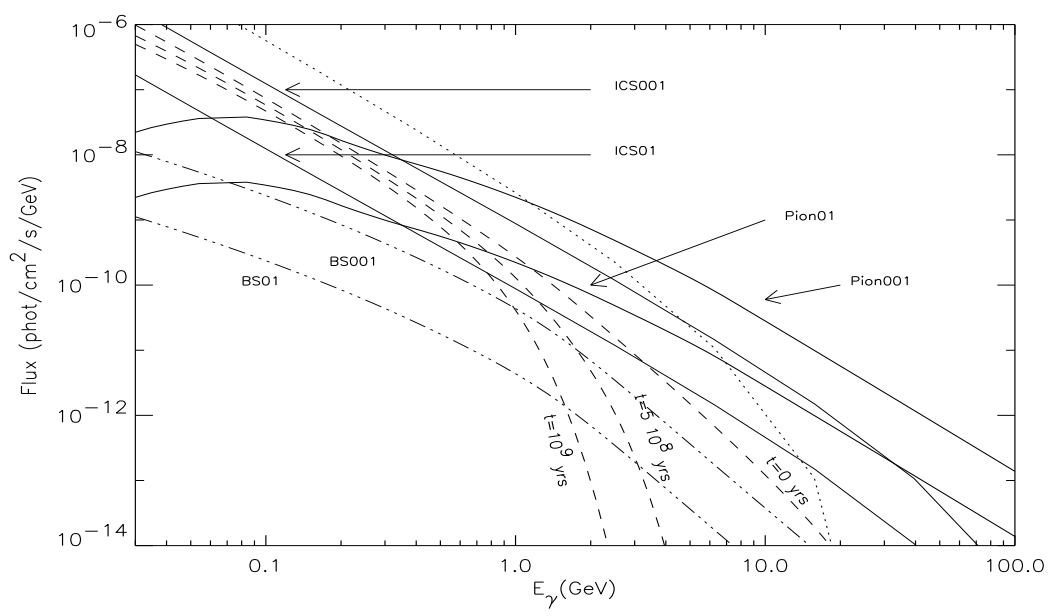

Figure 2. Predicted gamma ray emission from the Coma cluster (Blasi 2000). A detailed description of the curves can be found in the text. 
spectrum of the injected particles has been chosen to reproduce the spectral slope of the radio halo in Coma. The solid thick lines, labelled as Pion01 and Pion001 are the gamma ray fluxes due to neutral pion decay in $p p$ inelastic scattering. The normalization between electrons and protons accelerated at the same merger shock (therefore with the same power law injection spectrum in momentum) is parametrized by a parameter $\xi$ representing the ratio in the number density of electrons and protons at injection. After fixing $\xi$ the solution of the transport equation (including diffusion and energy losses) can be determined. For the numerical calculations we use $\xi=0.1$ (curve Pion01) and $\xi=0.01$ (curve Pion001). The thin solid lines labelled as ICS01 and ICS001 represent the gamma ray fluxes due to ICS of the secondary electrons from the decay of charged pions for the two values of the parameter $\xi$. The two curves labelled $B S 01$ and BS001 are the gamma ray fluxes due to bremsstrahlung emission of secondary electrons. The dashed lines are the result of bremsstrahlung emission of primary electrons, as a function of the time from the end of the merger event.

The dotted line is the flux of gamma rays due to ICS of the primary electrons. Note that this conctribution exists only for Bohm diffusion coefficient, as the acceleration time of electrons exceeds the timescale for losses for less extreme choices of the diffusion coefficient. In these more reasonable cases, the ICS emission ends up in the X-rays and stops there.

Some very general conclusions can be extracted from fig. 1:

i) The fluxes that are expected from a Coma-like cluster are accessible to GLAST in the energy region above $100 \mathrm{MeV}$.

ii) While the gamma ray emission from primary electrons is always time dependent and rapidly fading away after the end of the merger (it basically disappears a few hundred million years after the merger), the gamma ray emission due to either neutral pion decay or emission of secondary electrons is time independent (and actually slightly increasing with time), as a result of cosmic ray confinement and of slow energy losses of relativistic protons.

iii) The contribution due to protons is underestimated in fig. 2, because only one merger has been considered. While this is an excellent approximation for relativistic electrons, it is not for protons, which are accumulated during the cluster's history piling up to a likely larger value than that used to obtain the curves in fig. 2. Their spectrum depends however on the specific injection mechanism.

iv) Above a few $\mathrm{GeV}$ the gamma ray flux is likely to be dominated by the decay of neutral pions. The spectra are as flat as those of the parent protons and may extend to very high energies. In the energy region above a few hundred $\mathrm{GeV}$ these fluxes will be accessible to ground based experiments (see also fig. 1) such as VERITAS, MAGIC and HESS.

v) The ICS contribution of primary electrons is present only if electrons can attain a maximum Lorentz factor larger than about $10^{6}-10^{7}$, which may happen only for very small diffusion coefficients, like in the Bohm case 
(Blasi 2000). These considerations are crucial for models that try to establish a connection between cluster mergers and the diffuse extragalctic gamma ray background (Loeb \& Waxman 2000).

The main conclusions listed above remain valid even if the nonthermal particles are accelerated at a place other than merger shocks. Nevertheless, much interest has been shown recently on merger events as the origin of nonthermal phenomena in clusters of galaxies. Therefore in the next section, we discuss in detail the acceleration of particles in merger shocks, and their relevance on cosmological time scales.

\section{Merger shocks as cosmic ray accelerators}

Relativistic particles can be accelerated at strong shocks by diffusive (first order) Fermi acceleration (Fermi 1949; Blandford \& Eichler 1987). This mechanism has been invoked several times as the ideal acceleration process in clusters of galaxies that have been involved in a merger event (Blasi 2000; Fujita \& Sarazin 2001). In $\S 4.1$ we briefly summarize the basic physics of shock acceleration, since it is instrumental to understand whether merger related shock waves can indeed play a role for the acceleration of the relativistic particles responsible for the observed nonthermal radiation from clusters of galaxies. In order to assess this point, we also need to reconstruct the merger history of a cluster, and use it to determine the statistics of strengths of the shocks associated to the merger events. We do this in $\S 4.2$. The results summarized here are discussed at length by Gabici \& Blasi (2002).

\subsection{The basics of shock acceleration in clusters}

A shock with compression factor $r$ and Mach number $\mathcal{M}$ can accelerate particles to a power law in momentum $f(p) \propto p^{-\alpha}$, with slope $\alpha$ related to the Mach number and compression factor by the following expressions:

$$
\alpha=\frac{r+2}{r-1}=2 \frac{\mathcal{M}^{2}+1}{\mathcal{M}^{2}-1} .
$$

The acceleration occurs diffusively, in that particles scatter back and forth the shock, gaining at each crossing and recrossing an amount of energy proportional to the energy of the particle itself, $\Delta E / E \sim V / c$, where $V$ is the speed of the shock and $c$ is the speed of light. The distribution function of the accelerated particles is normalized here by $\int_{p_{\min }}^{p_{\max }} d p E(p) f(p)=\eta \rho u^{2}$, where $E(p)=\sqrt{p^{2}+m^{2}}$ and $m$ is the mass of the accelerated particles, $\eta$ is an efficiency of acceleration, $\rho$ and $u$ are the density and speed respectively of the fluid crossing the shock surface. The minimum and maximum momenta $\left(p_{\min }\right.$ and $\left.p_{\max }\right)$ of the accelerated particles are determined by the properties of the shock. In particular, $p_{\max }$ is the result of the balance between the acceleration rate and either the energy loss rate or the rate of escape from the acceleration region. Less clear is how to evaluate $p_{\text {min }}$; the minimum momentum of the particles involved in the acceleration process depends on the microphysics of the shock, a problem well known in the investigation of shock acceleration as the injection problem. Fortunately, most physical observables usually depend very weakly on $p_{\min }$. 
In the following we estimate the value of the maximum energies for electrons and protons as accelerated particles. The acceleration time, as a function of the particle energy $E$ can be written as

$$
\tau_{a c c}(E)=\frac{3}{u_{1}-u_{2}} D(E)\left[\frac{1}{u_{1}}+\frac{1}{u_{2}}\right]=\frac{3 D(E)}{u_{1}^{2}} \frac{r(r+1)}{r-1},
$$

valid for any choice of the diffusion coefficient $D(E)$, for which we consider two possible models. First we use the expression proposed in (Blasi \& Colafrancesco 1999):

$$
D(E)=2.3 \times 10^{29} B_{\mu}^{-1 / 3} L_{20}^{2 / 3} E(G e V)^{1 / 3} \mathrm{~cm}^{2} / s,
$$

where $B_{\mu}$ is the magnetic field in microgauss. Here we assumed that the magnetic field is described by a Kolmogorov power spectrum.

In this case the acceleration time becomes:

$$
\tau_{a c c}(E) \approx 6.9 \times 10^{13} B_{\mu}^{-1 / 3} L_{20}^{2 / 3} E(G e V)^{1 / 3} v_{8}^{-2} g(r) \quad s,
$$

where $v_{8}=\frac{v}{10^{8} \mathrm{~cm} / \mathrm{s}}$ and $g(r)=r(r+1) /(r-1)$ and $v=u_{1}$.

For electrons, if the average magnetic field is less than $\sim 3 \mu G$, the energy losses are dominated by ICS off the microwave background, with a loss time $\tau_{\text {loss }} \approx 4 \times 10^{16} / E s$, where $E$ is in $\mathrm{GeV}$. The maximum energy of accelerated electrons is obtained requiring $\tau_{\text {acc }}<\tau_{\text {loss }}$ :

$$
E_{\text {max }}^{e} \approx 118 L_{20}^{-1 / 2} B_{\mu}^{1 / 4} v_{8}^{3 / 2} g(r)^{-3 / 4} \mathrm{GeV} .
$$

The compression ratio $r$ and the velocity $v_{8}$ are not independent, since

$$
r=\frac{\frac{8}{3} \mathcal{M}^{2}}{\frac{2}{3} \mathcal{M}^{2}+2},
$$

valid for an ideal monoatomic gas.

For protons, energy losses are not relevant and the maximum energy is clearly determined by the finite time duration of the merger event. Therefore the maximum energy for protons will be defined by the condition $\tau_{a c c}<t_{\text {merger }}$, which gives

$$
E_{\text {max }}^{p} \approx 9 \times 10^{7} L_{20}^{-2} B_{\mu} v_{8}^{6} g(r)^{-1 / 2} \mathrm{GeV} .
$$

As a second possibility for the diffusion coefficient we assume Bohm diffusion, well motivated for the case of strong turbulence. In this case:

$$
D(E)=3.3 \times 10^{22} E(G e V) / B_{\mu} \mathrm{cm}^{2} / s .
$$

In this case, for electrons we obtain:

$$
E_{\text {max }}^{e} \approx 6.3 \times 10^{4} B_{\mu}^{1 / 2} v_{8} g(r)^{-1 / 2} \mathrm{GeV},
$$

while for protons

$$
E_{\text {max }}^{p} \approx 3 \times 10^{9} B_{\mu} v_{8}^{2} g(r)^{-1} \mathrm{GeV} .
$$

If $E_{\text {max }}^{p}$ becomes larger than $\sim 10^{10} \mathrm{GeV}$ energy losses due to pair production and photopion production on the photons of the microwave background become important and limit the maximum energy to less that a few $10^{10} \mathrm{GeV}$. 


\subsection{The merger tree and related shocks}

The standard theory of structure formation predicts that larger structures are the result of the mergers of smaller structures: this hierarchical model of structure formation in the universe has been tested in several independent ways and provides a good description of the observations of the mass function of clusters of galaxies and their properties.

While a complete understanding of the process of structure formation can only be achieved by numerical N-body simulations, an efficient and analytical description can also be obtained and several of these approaches are widely discussed in the literaure. Historically, the first approach to the problem was proposed by Press \& Schechter (1974, hereafter PS) and successively developed by Bond et al. (1991) and Lacey \& Cole (1993, hereafter LC) among others. In the PS formalism, the differential comoving number density of clusters with mass $M$ at cosmic time $t$ can be written as:

$$
\frac{d n(M, t)}{d M}=\sqrt{\frac{2}{\pi}} \frac{\varrho}{M^{2}} \frac{\delta_{c}(t)}{\sigma(M)}\left|\frac{d \ln \sigma(M)}{d \ln M}\right| \exp \left[-\frac{\delta_{c}^{2}(t)}{2 \sigma^{2}(M)}\right] .
$$

The rate at which clusters of mass $M$ merge at a given time $t$ is written as a function of $t$ and of the final mass $M^{\prime}$ (LC, 1993):

$$
\begin{aligned}
& R\left(M, M^{\prime}, t\right) d M^{\prime}= \\
& \sqrt{\frac{2}{\pi}}\left|\frac{d \delta_{c}(t)}{d t}\right| \frac{1}{\sigma^{2}\left(M^{\prime}\right)}\left|\frac{d \sigma\left(M^{\prime}\right)}{d M^{\prime}}\right|\left(1-\frac{\sigma^{2}\left(M^{\prime}\right)}{\sigma^{2}(M)}\right)^{-3 / 2} \\
& \exp \left[-\frac{\delta_{\mathrm{c}}^{2}(\mathrm{t})}{2}\left(\frac{1}{\sigma^{2}\left(\mathrm{M}^{\prime}\right)}-\frac{1}{\sigma^{2}(\mathrm{M})}\right)\right] \mathrm{dM}^{\prime},
\end{aligned}
$$

where $\varrho$ is the present mean density of the universe, $\delta_{c}(t)$ is the critical density contrast linearly extrapolated to the present time for a region that collapses at time $t$, and $\sigma(M)$ is the current rms density fluctuation smoothed over the mass scale $M$. For $\sigma(M)$ we use an approximate formula proposed by Kitayama (1997), normalized by assuming a bias parameter $b=0.9$. We adopt the expression of $\delta_{c}(t)$ given by Nakamura \& Suto (1997). In this respect our approach is similar to that adopted by Fujita \& Sarazin(2001).

Salvador-Solé, Solanes \& Manrique (1998) modified the model illustrated above, by introducing a new parameter, $\Delta_{m}=r_{c r i t}=\left[\left(M^{\prime}-M\right) / M\right]_{\text {crit }}$, defined as a peculiar value of the captured mass that separates the accretion events from

merger events. Events in which a cluster of mass $M$ captures a dark matter halo with mass smaller then $\Delta_{m} M$ are considered as continuous mass accretion, while events where the collected mass is larger than $\Delta_{m} M$ are defined as mergers. The value of $\Delta_{m} M$ is somehow arbitrary.

Using this effective description of the merger and accretion events, it is easy to construct simulated merger trees for a cluster with fixed mass at the present time. Although useful from a computational point of view, this difference does not correspond to any new phyics information, therefore in the following we will adopt the name "merger" for both regimes, provided there is no ambiguity or 


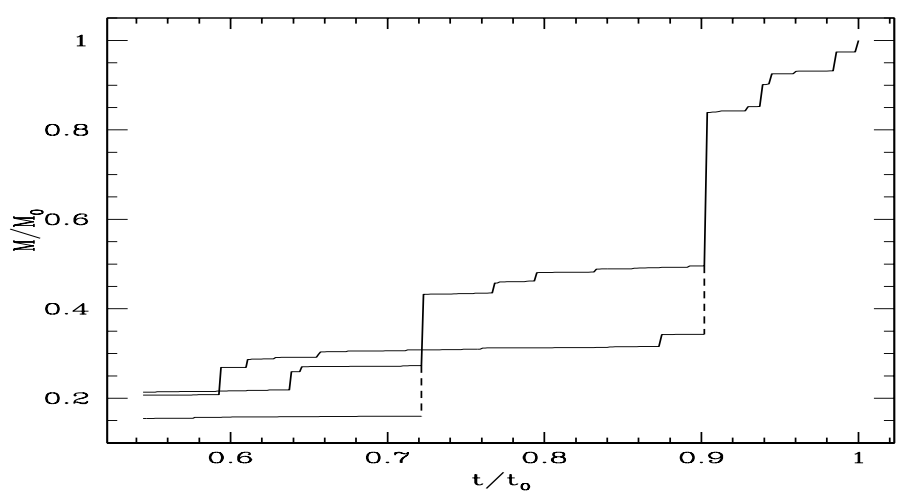

Figure 3. Merger history of a cluster with present mass $10^{15}$ solar masses (Gabici \& Blasi 2002). The mass (y-axis) suffers major jumps in big merger events. Time is on the x-axis.

risk of confusion. In figure 3 we plotted the merger tree for a cluster with present mass equal to $10^{15} M_{\odot}$ and for $\Delta_{m}=0.6$. The big jumps in the cluster mass correspond to merger events, while smaller jumps correspond to what SalvadorSolé et al. (1998) defined as accretion events.

While the dark matter components of two clusters involved in a merger can compenetrate each other due to the collisionless nature of dark matter, and form a deeper gravity potential well, the baryon components of the clusters are forced to move supersonically during the merger event, and shock surfaces are formed. These shocks are instrumental for the heating of the intracluster gas, since they allow the conversion of part of the available gravitational energy into thermal energy.

In this section we describe in more detail the physical properties of such shocks, with special attention for their Mach numbers and compression factors.

We assume to have two clusters, as completely virialized structures, at temperatures $T_{1}$ and $T_{2}$, and with masses $M_{1}$ and $M_{2}$ (here the masses are the total masses, dominated by the dark matter component). The virial radius of a cluster can be written as follows

$$
\begin{gathered}
r_{v i r, i}=\left(\frac{3 M_{i}}{4 \pi \Delta_{c} \rho_{c r, 0}\left(1+z_{f, i}\right)^{3}}\right)^{\frac{1}{3}}= \\
\left(\frac{G M_{i}}{100 \Omega_{m} H_{0}^{2}\left(1+z_{f, i}\right)^{3}}\right)^{\frac{1}{3}},
\end{gathered}
$$

where $i=1,2, \rho_{c r, 0}=\Omega_{m} 1.8810^{-29} h^{2} \mathrm{~g} \mathrm{~cm}^{-2}$ is the current value of the critical mass density of the universe, $z_{f, i}$ is the redshift of formation of the cluster $i$, $\Delta_{c}=200$ is the density constrast for the formation of the cluster and $\Omega_{m}$ is the matter density fraction. In the right hand side of the equation we used the fact that $\rho_{c r, 0}=3 H_{0}^{2} / 8 \pi G$, where $H_{0}$ is the Hubble constant. The formation redshift $z_{f}$ is on average a decreasing function of the mass, meaning that smaller clusters are formed at larger redshifts, consistently with the hierarchical scenario of structure formation. There are intrinsic fluctuations in the value of $z_{f}$ from cluster to cluster at fixed mass, due to the stochastic nature of the merger tree. 
Two clusters with masses $M_{1}$ and $M_{2}$ collide with a relative velocity $V_{r}$ that can be easily calculated from energy conservation:

$$
-\frac{G M_{1} M_{2}}{r_{v i r, 1}+r_{v i r, 2}}+\frac{1}{2} M_{r} V_{r}^{2}=-\frac{G M_{1} M_{2}}{2 R_{12}},
$$

where $M_{r}=M_{1} M_{2} /\left(M_{1}+M_{2}\right)$ is the reduced mass and $R_{12}$ the turnaround radius of the system of two masses. In a Einstein-De Sitter cosmology, the latter equals twice the virial radius of the system, so that, using eq. 15, we get:

$$
R_{12}=\left(\frac{M_{1}+M_{2}}{M_{1}}\right)^{1 / 3} r_{v i r, 1}
$$

In different cosmologies this expression still remains valid in approximate way. The sound speed of the cluster $i$ is given by

$$
c_{s, i}=\gamma_{g}\left(\gamma_{g}-1\right) \frac{G M_{i}}{2 r_{v i r, i}}
$$

where we used the virial theorem to relate the gas temperature to the mass and virial radius of the cluster. The adiabatic index of the gas is $\gamma_{g}=5 / 3$. Following Takizawa (1999), the Mach numbers of each cluster while moving in the volume of the other cluster can be written as:

$$
\begin{aligned}
\mathcal{M}_{1}^{2} & =\frac{4(1+\eta)}{\gamma(\gamma-1)}\left[\frac{1}{1+\frac{1+z_{f, 1}}{1+z_{f, 2}} \eta^{1 / 3}}-\frac{1}{4 \frac{1+z_{f, 1}}{1+z_{f}}(1+\eta)^{1 / 3}}\right] \\
\mathcal{M}_{2}^{2} & =\eta^{-2 / 3} \frac{1+z_{f, 1}}{1+z_{f, 2}} \mathcal{M}_{1}^{2}
\end{aligned}
$$

where $\eta=M_{2} / M_{1}<1$ and $z_{f}$ is the formation redshift of the cluster with mass $M_{1}+M_{2}$. Our strategy at this point is to consider a cluster with mass $M_{0}$ at the present time and simulate numerous merger trees and calculate the Mach numbers of the subclusters taking part to the merger events. To start with, we simulate 500 realizations of the merger history of a $10^{15} M_{\odot}$ cluster. A value $\Delta_{m}=0.05$ is assumed, much lower than in (Fujita \& Sarazin 2001). This simply implies that we follow the histories of very small halos of dark matter, rather than the big ones only. The results of our calculations of the Mach numbers are plotted in fig. 4a (left panel). It is evident from the figure that for major mergers, involving clusters with comparable masses $(\eta \sim 1)$, the Mach numbers of the shocks are of order unity. In other words the shocks are only moderately supersonic. In order to achieve Mach numbers of order of $3-4$ it is needed to consider mergers between clusters with very different masses $(\eta \sim 0.05)$, which, in the language of Salvador-Solé et al. (1998) and Fujita \& Sarazin (2001) would not be considered as mergers but rather as continuous accretion. These events are the only ones that produce strong shocks, and this is of crucial importance for the acceleration of suprathermal particles, as discussed below. This picture seems to be confirmed by X-ray observations (see for instance Markevitch, Sarazin and Vikhlinin (1999)). 

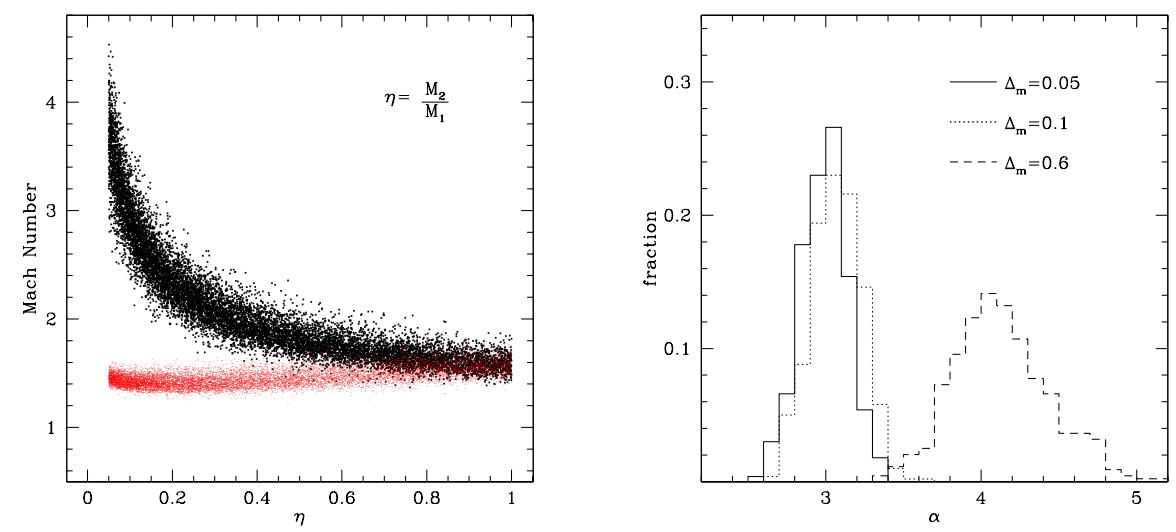

Figure 4. $\quad 4 a$ (left panel): Distribution of the Mach numbers of merger related shocks as a function of the mass ratio of the merging subclusters. The upper strip is the distribution of Mach numbers in the smaller cluster, while the lower strip refers to the bigger cluster. $4 b$ (right panel): Slope of the time-integrated proton spectrum resulting from all the mergers in a cluster. The three curves are obtained for $\Delta_{m}=0.05$ (solid line), $\Delta_{m}=0.1$ (dotted line) and $\Delta_{m}=0.6$ (dashed line).

Fig. 4b (right panel) requires some preliminary comments: during a merger event, new particles are accelerated from the thermal pool, but the protons already confined in the cluster volume are also reaccelerated. These processes are taken into account in detail by Gabici \& Blasi (2002). The final spectra of protons at the present time, as a result of the superposition of all the merger events that a cluster suffered, have a shape which can be something more complicated than a simple power law (Gabici \& Blasi, 2002), therefore we choose here to plot, in fig. 4b, the slope at fixed energy, say $10 \mathrm{GeV}$ (one should remember that this corresponds to secondary electrons with typical energy of a few GeV). The result of our calculations show that the time integrated proton spectra are typically steep, or at least steeper than needed to explain the observed nonthermal radiation, even in the cases $\Delta_{m} \ll 1$.

The situation is slightly different for primary electrons. High energy electrons must be relatively young to generate appreciable nonthermal radiation. A typical time for the production of these electrons is of about one billion years. In other words, only electrons injected in the last few mergers (or accretion events) can generate nonthermal radiation at present. Hence, we generated a merger tree of a cluster with Coma-like mass and extended it only for one billion years in the past, and again calculated the spectra of the electrons accelerated at the merger shocks. We repeated this procedure for 500 clusters and only about $30 \%$ of them suffered any kind of merger (down to $\eta=0.05$ ) in the last billion years. Of these, $\sim 20 \%$ are characterized by strong shocks, able to accelerate particles with spectra flatter than $E^{-2.4}$. In other words, only $\sim 6 \%$ of clusters with mass comparable with the Coma cluster should have a similar radio halo. It is 
important to stress again that strong shocks are not associated to major mergers but rather to accretion events.

\section{The extragalactic diffuse gamma ray background}

Since the detection of an isotropic excess in the gamma ray emission of the Galaxy, as detected by EGRET (Sreekumar et al. 1998), and interpreted as of extragalactic origin, several attempts have been made of relating it to nonthermal processes occurring in clusters of galaxies. The initial paper by Dar \& Shaviv (1995) reached incorrect conclusions, mainly due to an errouneous calculation of the spectrum of the radiation, as later recognized by Berezinsky, Blasi \& Ptuskin (1997). More recently, Loeb \& Waxman (2000) have reproposed this connection: in their paper the gamma ray emission is generated by ICS of relativistic electrons accelerated at the shocks generated during structure formation. This scenario has received much attention and also inspired some searches for associations between the EGRET unidentified sources and the positions of some clusters of galaxies (Colafrancesco 2002; Kawasaki \& Totani 2001, Totani \& Kitayama 2000).

Some of the shocks related to the formation of large scale structures are related to cluster mergers, in that they form within the virial radii of the merging clusters. Other shocks form in the outer regions and propagate in a colder medium, therefore reaching higher Mach numbers (Miniati, F., et al. 2000). The Mach numbers of the shocks related to structure formation range between unity and a few hundreds. The weaker shocks, usually associated to major mergers, as discussed by Gabici \& Blasi (2002) are inefficient particle accelerators (see also the discussion in $§ 4.2$ ). The stronger shocks extend over several Mpc regions and are strong enough to generate flat spectra. To understand whether the former or the latter dominate, it is needed to run careful numerical simulations, as performed by Miniati (2002). One point that should be made clear is that the possibility that part of the diffuse extragalactic gamma ray background may have a connection to large scale structures relies upon the strong assumption that the diffusion coefficient of the particles around the shock surface is well described by a Bohm diffusion coefficient. Only in this case the electron energies may be large enough to generate gamma rays by ICS off the photons of the cosmic microwave backgound. Moreover, the magnetic fields that have been estimated from the observations of nonthermal radiation in clusters are of fraction of $\mu G$, but they only refer to the virialized region of the clusters. In the flux freezing approximation, the magnetic field scales with the radial coordinate as $B \sim$ $\rho^{2 / 3} \sim r^{-3 \beta}$, where $\beta \approx 0.75$ is the parameter entering the $\beta$-model for the density $\rho$ (simulations performed by Dolag, Bartelmann \& Lesch (2002) show even steeper trends). At the distance of the large Mach number shocks, say comparable to the turnaround radius of a cluster, the strength of the field is likely reduced to $n G$ values. For a typical scale of $5 \mathrm{Mpc}$, the maximum energy of accelerated electrons, as derived in eq. 11, is reduced to values that are barely sufficient for the production of gamma rays up to $\sim 10-30 \mathrm{GeV}$, even in the case of Bohm diffusion. Although there are several aspects that deserve further investigation, the possibility that at least a fraction of the alleged extragalactic gamma ray background may be due to processes related to structure formation 
is certainly interesting and will continue to fuel much interest in the years to come.

\section{Conclusions}

The diffuse medium in the intracluster volume is filled with a nonthermal gas of particles that are the relics of all the events occurred within the cluster itself. Gamma ray astronomy is an important tool to study this component and infer information about particle acceleration and confinement and about the specific processes (mergers, active galactic phases, and many others) that inject nonthermal particles in a cluster and possibly contribute to its heating.

We discussed here the expectations for gamma ray fluxes in the presence of both protons and primary electrons in clusters. Even if the amount of hadronic cosmic rays trapped on cosmological scales is, say, $10 \%$ of the equipartition energy, we expect that the gamma ray fluxes may be detectable by GLAST for energies above $100 \mathrm{MeV}$, and by future ground based gamma ray telescopes such as VERITAS, MAGIC and HESS at higher energies. Even current observations with STACEE and HEGRA could actually provide interesting information about the abundance of cosmic rays in the intracluster gas of nearby clusters, as shown in fig. 1. Unfortunately, we are not aware of any scientific report of such attempt to look for high energy gamma rays with either STACEE or HEGRA. While the higher energy gamma ray emission is likely to be generated by hadronic interactions, the lower energy gamma rays (in the $\mathrm{MeV}-\mathrm{GeV}$ range) can be generated by several processes related to electrons, and most of the fluxes derived in the literature are in the range of interest for GLAST.

The importance of gamma ray observations can be appreciated particularly well in the context of the growing multifrequency observations, that one piece at a time, are allowing us to understand the processes that occur in the intracluster volume, enriching it with hot gas, nonthermal particles and magnetic fields, in a way that at present is still unclear.

\section{References}

Berezinsky, V. S., Blasi, P., \& Ptuskin, V. S. 1997, ApJ, 487, 529

Blandford, R., \& Eichler, D. 1987, Phys Rep, 154, 1

Blasi, P., Brunetti, G., \& Gabici, S., in preparation

Blasi, P., \& Colafrancesco, S. 1999, Astropart. Phys., 12, 169

Blasi, P., 1999, ApJ, 525, 603

Blasi, P. 2000, ApJ, 532, L9

Blasi, P. 2001, Astropart. Phys., 15, 223

Bond, J. R., Cole, S., Efstathiou, G., \& Kaiser, N. 1991, ApJ, 379, 440

Brunetti, G., Setti, G., Feretti, L., \& Giovannini, G. 2001a, MNRAS, 320, 365

Brunetti, G., Setti, G., Feretti, L., \& Giovannini, G. 2001b, NewA, 6, 1

Clarke, T.E., Kronberg, P.P., \& Böringer, H., in the proceedings of the Ringberg Workshop "Diffuse Thermal and Relativistic Plasma in Galaxy Clusters", Eds: H. Böringer, L. Feretti, P. Schuecker 
Colafrancesco, S., preprint astro-ph/0203443

Colafrancesco, S., Blasi, P., 1998, Astropart. Phys., 9, 227

Dar, A., \& Shaviv, N.J., 1995, Phys. Rev. Lett., 75, 3052

Dennison, B., 1980, ApJ, 239, 93

Dogiel, V.A., 2000, A\&A, 357, 66

Eilek, J.A., 1999, in the proceedings of the Ringberg Workshop "Diffuse Thermal and Relativistic Plasma in Galaxy Clusters", Eds: H. Böringer, L. Feretti, P. Schuecker

Ensslin, T.A., Lieu, R., \& Biermann, P.L., 1999, A\&A, 344, 409

Feretti, L., Brunetti, G., Giovannini, G., Govoni, F., \& Setti, G. 2000, in IAP 2000 meeting, Constructing the Universe with Clusters of Galaxies, ed. F. Durret, \& D. Gerbal

Fermi, E. 1949, Phys. Rev., 8, 1169

Fujita, Y., \& Sarazin, C. L. 2001, ApJ, 563, 660

Fusco-Femiano, R., Dal Fiume, D., Feretti, L., Giovannini, G., Grandi, P., Matt, G., Molendi, S., \& Santangelo, A. 1999, ApJ, 513, L21

Fusco-Femiano, R., et al. 2000, ApJ, 534, L7

Gabici, S., \& Blasi, P., in preparation.

Kawasaki, W., \& Totani, T., preprint astro-ph/0108309

Kitayama, T. 1997, Cosmological Implications of the Abundances of Clusters of Galaxies: PhD Thesis (The University of Tokyo)

Lacey, C., \& Cole, S. 1993, MNRAS, 262, 627

Lieu, R., Mittaz, J. P. D., Bowyer, S., Breen, J. O., Lockman, F. J., Murphy, E. M., \& Hwang, C.-Y. 1996, Science, 274, 1335

Loeb, A., \& Waxman, E., 2000, Nature 405, 156

Markevitch, M., Sarazin, C. L., \& Vikhlinin, A. 1999, ApJ, 521, 526

Miniati, F., preprint astro-ph/0203014

Miniati, F., Jones, T.W., Kang, H., \& Ryu, D., 2001, ApJ, 562, 233

Miniati, F., Ryu, D., Kang, H., Jones, T.W., Cen, R., \& Ostriker, J.P., 2000, ApJ, 542, 608

Nakamura, T. T., \& Suto, Y. 1997, Prog. Theor. Phys., 97, 49

Petrosian, V. 2001, ApJ, 557, 560

Press, W. H., \& Schechter, P. 1974, ApJ, 187, 425

Salvador-Solé, E., Solanes, J. M., \& Manrique, A. 1998, ApJ, 499, 542

Sarazin, C.L., Kempner, J.C., 2000, ApJ, 533, 73

Sreekumar, P. et al., 1996, ApJ, 464, 628

Sreekumar, P. et al., 1998, ApJ, 494, 523

Takizawa, M., 1999, ApJ, 520, 514

Totani, T., \& Kitayama, T., 2000, ApJ545, 572

Volk, H. J., Aharonian, F. A., \& Breitschwerdt, D. 1996, Space Sci. Rev., 75, 279 